MEASUREMENT

OF NON-ELECTRIC QUANTITIES

\title{
POSITION-SENSITIVE PHOTODETECTOR ARRAY FOR OPTICAL COORDINATOR
}

\author{
Volodimir Verbitskiy, Dr.Sc., Prof.; Andriy Voronko, Researcher; Dmytro Verbitskiy, Master; \\ National Technical University of Ukraine "Igor Sikorsky Kyiv Polytechnic Institute", Ukraine
}

\begin{abstract}
New position-sensitive matrices for an optical coordinator and a method for determining the coordinates of a light spot for micromovements are presented, an analysis of the topologies and direction-finding characteristics of the proposed matrices is shown.
\end{abstract}

Key words: Optical coordinator, Position-sensitive matrix, Method for determining coordinates, Position-sensitive detectors, Quadrant photodiodes, Direction-finding characteristics.

\section{Introduction}

Precision optical coordinators are widely used to determine micromovements. The main task of the optical coordinator is to precisely determine the spatial position of the light spot, usually formed by a laser diode or LED with collimating optics and the corresponding radiation spectrum. Usually, such systems use one- or twodimensional position-sensitive photodetectors (PSD): CCD; active pixel sensors (APS), which include CMOS Image Sensors (CIS) [1-4]; photodiodes based on the lateral effect [5-9]. All of the above types of detectors have several technical disadvantages (low speed, sensitivity, and accuracy, exposure to light), which in some cases, restrict their use. It is better to use quadrant photodiodes (QPD) for the precise determination of the coordinates of the light spot [10-13].

\section{Formulation of the problem}

The 4-quadrant photodetector has a good resolution of the position of the light spot (high steepness of the direction-finding characteristic) in a relatively small range (movement does not exceed the size of the radius of the light spot) relative to the center. Therefore, the functional diagram of the optical coordinator, based on a 4-quadrant photodetector, must necessarily include a tracking system. Precision tracking systems are specially used to maintain the light spot in the center of the fourquadrant photodiode. Optical coordinators of this type are used in many measuring systems, where it is necessary to very accurately determine the coordinates of a light spot. For example, the optical coordinator for determining the coordinates of a reflected beam from a cantilever in an atomic force microscope is arranged according to this scheme. To increase the capture area of the tracking system, it is necessary to form a light spot of a larger diameter. At the same time, the steepness of the direction-finding characteristic (dependence of the amplitude of the output signals of the photosensitive elements of the position-sensitive photodetector from the position of the light spot on it) and, consequently, holding accuracy of the light spot in the nominal position depends on the size ratio between light spot and quadrants of the photodetector. Reducing the diameter of the light spot increases the measurement resolution of the optical coordinator. In this case, it is necessary to use the more precise $\mathrm{X}-\mathrm{Y}$ micromanipulators for the tracking system of the optical coordinator. Thus, providing both a large width of the capture area and high accuracy of determining the coordinates of a light spot at the same time are contradictory requirements that cannot be realized using a four-quadrant photodetector as a positionsensitive element. For this reason, these optical coordinators always require an initial precision alignment of the light beam position.

In some cases, extremely high requirements are imposed on the dynamic range of optical coordinators, which are practically unattainable with known construction schemes. In known schemes for constructing optical coordinators, the optical system focuses radiation into a spot whose size is smaller than one photosensitive element. It turns out that at the required minimum level of the optical signal it is impossible to meet the requirements for the signal-to-noise ratio, and at a given level of the maximum optical signal the photosensitive elements of the matrix are destroyed.

\section{The goal of the work}

The purpose of this work is to develop the positionsensitive photodetector array for an optical coordinator with a large capture area and high accuracy in determining the position of the light spot, if possible with a minimum number of processed signals from the position-sensitive element, i.e. essentially creating a precision optical coordinator without tracking system and an initial alignment of the light beam. It is also necessary to develop a photosensitive matrix with an extended dynamic range for measurement the position of a radiation source with an optical signal not normalized in power. 


\section{Photosensitive array topology}

To create an optical coordinator without a tracking system, it is necessary to resolve the contradiction between the accuracy of determining the position of the light spot and the capture area of the optical coordinator. To solve this problem, a new method is proposed for determining the coordinates of the light spot and using a position-sensitive matrix of photosensitive elements with a special topology of photosensitive elements interconnections, which simultaneously provides both a high slope of the direction-finding characteristic and a wide capture zone of the tracking system. According to the proposed topology, the photosensitive elements are arranged in rows and columns like a "checkerboard", and the photosensitive elements located on the "white" fields are connected into columns, and those located on the "blac" fields - into rows (Fig. 1).

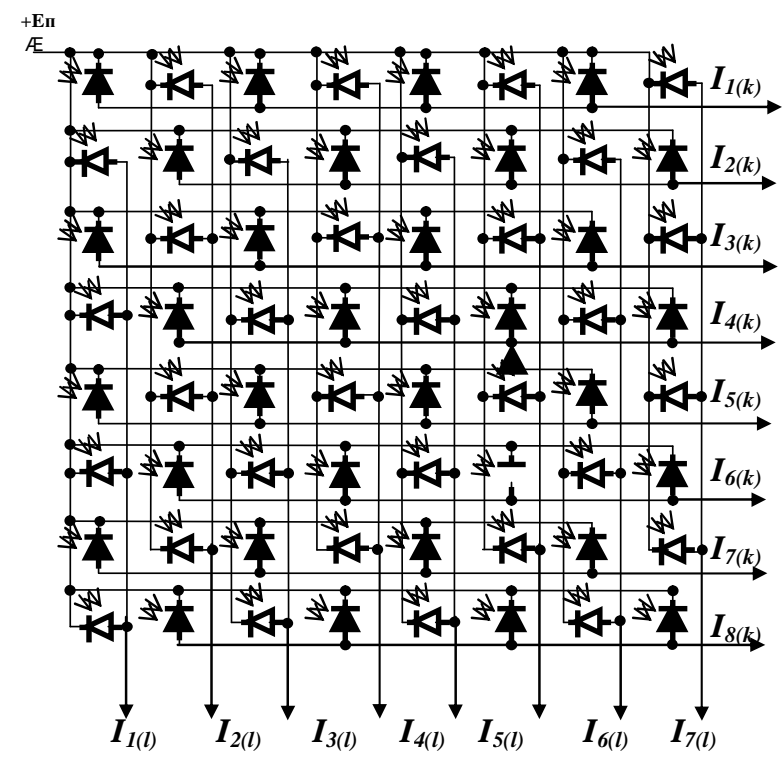

Fig. 1. Diagram of a position-sensitive matrix of photosensitive elements.

Photocurrents of elements of each row (column) located after one are summed up. Thus, at the outputs of the proposed matrix signals are generated equal to the sum of photocurrents from the elements of the corresponding rows (columns) of this matrix:

$$
\begin{gathered}
I_{2 k-1}=\sum_{l=1}^{\frac{n}{2}} I_{(2 k-1),(2 l-1)}, \\
I_{2 k}=\sum_{l=1}^{\frac{n}{2}} I_{2 k, 2 l}, \\
I_{2 l-1}=\sum_{k=1}^{\frac{m}{2}} I_{2 k,(2 l-1)}, \\
I_{2 l}=\sum_{k=1}^{\frac{m}{2}} I_{(2 k-1), 2 l},
\end{gathered}
$$

where $\mathrm{m}$ is the number of the matrix rows; $\mathrm{n}$ is the number of the matrix columns;

$$
k=1,2,3, \ldots, \frac{m}{2} \quad l=1,2,3, \ldots, \frac{n}{2}
$$

Note, that in such a matrix the number of rows and columns is not limited by the requirement of multiplicity 4 , but can be arbitrary.

\section{Analysis of direction-finding characte-} ristics

By the direction-finding characteristics of the optical coordinator, we mean the set of dependencies of the electrical signals FEi and FEj from the outputs of the position-sensitive matrix of photosensitive elements with a special topology of interconnections, based on which the coordinator is built, on the angular coordinates $\alpha, \beta$ of the beam incident on the matrix:

$$
\left\{\begin{array}{cc}
F E i_{1}(\alpha, \beta) ; & F E j_{1}(\alpha, \beta) ; \\
F E i_{2}(\alpha, \beta) ; & F E j_{2}(\alpha, \beta) ; \\
\cdots & \cdots \\
F E \dot{i}_{N}(\alpha, \beta) ; & F E j_{N}(\alpha, \beta)
\end{array}\right\}
$$

where FEi is the electrical signal from the output, which corresponds to the $\mathrm{i}$-th row of the matrix; FEj is an electrical signal from the output, which corresponds to the $\mathrm{j}$-th column of the matrix; $\mathrm{N}$ is the number of electrical signals from the matrix outputs, which depends on the size of the light spot formed by the optical system of the coordinator in the direction-finding dependences is $2 \mathrm{~N}$.

Since the angular coordinates $\alpha, \beta$ (in two orthogonal planes) of the plane of the photosensitive areas of the matrix (normalized to the half-period of the matrix). The total number of the incident beam by the transfer function $\mathrm{K}$ of the optical scheme of the coordinator are associated with the corresponding linear coordinates $\mathrm{x}$, $\mathrm{y}$ of the center $\mathrm{O}$ of the light spot formed in the plane of the matrix:

$$
\left\{\begin{array}{l}
x_{O}=K \cdot \alpha \\
y_{O}=K \cdot \beta
\end{array}\right.
$$

The direction-finding characteristics FEi and FEj of the optical coordinator are completely determined by the corresponding direction-finding characteristics FSi and $\mathrm{FSj}$ of the position-sensitive matrix:

$$
\left\{\begin{array}{cc}
F S i_{1}\left(x_{O}, y_{O}\right) ; & F S j_{1}\left(x_{O}, y_{O}\right) ; \\
F S i_{2}\left(x_{O}, y_{O}\right) ; & F S j_{2}\left(x_{O}, y_{O}\right) ; \\
\cdot \ldots & . . \\
F S i_{N}\left(x_{O}, y_{O}\right) ; & F S j_{N}\left(x_{O}, y_{O}\right)
\end{array}\right\}
$$

Here the FSi is the total electrical signal from the output of the matrix corresponding to its i-th row; FSj is the total electrical signal from the output corresponding to its $j$-th column; $x$ is the linear coordinate of the center of the light spot $\mathrm{O}$ along the axis perpendicular to the direction of the matrix rows; $y$ is the linear coordinate of the center of the light spot $\mathrm{O}$ along the axis perpendicular to the direction of the matrix columns.

In this case, it is sufficient to determine the direction-finding characteristics FSi and FSj of the position-sensitive matrix on the interval in the matrix plane corresponding to a square with a half-period size $d$ 
of the matrix for the coordinate plane Oxy with the origin at the center of the matrix element:

$$
\begin{gathered}
\left\{\begin{array}{l}
x_{O} \in\left[-\frac{d}{2} ;+\frac{d}{2}\right] ; \\
y_{O} \in\left[-\frac{d}{2} ;+\frac{d}{2}\right] .
\end{array} \begin{array}{l}
\text { position-sensitive matrix of photosensitive elements with } \\
\text { a special topology of interconnections are manifested in } \\
\text { the following properties: }
\end{array}\right. \\
{\left[\begin{array}{cc}
F S i_{I}\left(x_{O}+d, y_{O}\right)=F S j_{J}\left(y_{O}, x_{O}\right) ; & F S j_{J}\left(x_{O}+d, y_{O}\right)=F S i_{I-1}\left(y_{O}, x_{O}\right) ; \\
\left.F S i_{I}\left(x_{O}, y_{O}+d\right)\right)=F S j_{J-1}\left(y_{O}, x_{O}\right) ; & \left.F S j_{J}\left(x_{O}, y_{O}+d\right)\right)=F S i_{I}\left(y_{O}, x_{O}\right) ; \\
F S i_{I}\left(x_{O}+d, y_{O}+d\right)=F S i_{I-1}\left(x_{O}, y_{O}\right) ; & F S j_{J}\left(x_{O}+d, y_{O}+d\right)=F S j_{J-1}\left(x_{O}, y_{O}\right)
\end{array}\right.} \\
{\left[\begin{array}{cc}
F S i_{I}\left(x_{O}+2 d, y_{O}\right)=F S i_{I-2}\left(x_{O}, y_{O}\right) ; & F S j_{J}\left(x_{O}+2 d, y_{O}\right)=F S j_{J}\left(x_{O}, y_{O}\right) ; \\
\left.F S i_{I}\left(x_{O}, y_{O}+2 d\right)\right)=F S i_{I}\left(x_{O}, y_{O}\right) ; & \left.F S j_{J}\left(x_{O}, y_{O}+2 d\right)\right)=F S j_{J-2}\left(x_{O}, y_{O}\right) ; \\
F S i_{I}\left(x_{O}+2 d, y_{O}+2 d\right)=F S i_{I-2}\left(x_{O}, y_{O}\right) ; & F S j_{J}\left(x_{O}+2 d, y_{O}+2 d\right)=F S j_{J-2}\left(x_{O}, y_{O}\right)
\end{array}\right.}
\end{gathered}
$$

where I is the row number of the matrix element on which the center of the light spot is located with coordinates $\mathrm{x}, \mathrm{y} ; \mathrm{J}$ is the column number of the matrix element on which the center of the light spot with coordinates $\mathrm{x}, \mathrm{y}$ is located.

The optical signal on each matrix element is the luminous flux incident on the photosensitive area of the element, proportional to the area of the illuminated area of this element. The electrical signal-response from each photosensitive element of the matrix is proportional to the optical signal arriving at it. Photocurrent $\mathrm{I}_{\mathrm{i}, \mathrm{j}}$ from each matrix element is determined by the integral:

$$
I_{i, j}=k \int_{y 1_{i j}}^{y 2_{i j}} \int_{x 1_{i j}}^{x 2_{i j}} E(x, y) d x d y
$$

Here $i, j$ is the address of the matrix element due to the row number and column number; $\mathrm{k}$ is the coefficient of proportionality (current photosensitivity); $\mathrm{E}(\mathrm{x}, \mathrm{y})$ is an illumination in the spot; $\mathrm{x} 1 \mathrm{i}, \mathrm{j}, \mathrm{x} 2 \mathrm{i}, \mathrm{j}$ are the coordinates of the left and right boundaries of the photosensitive area $i, j$ of the element; $y 1 i, j, y 2 i, j$ are the coordinates of the upper and lower boundaries of the photosensitive area $i, j$ of the element.

In the mathematical model, we consider a matrix of photosensitive elements of a square shape of size $a \times a$ with gaps of width $t$ between them, on the surface of which an optical signal arrives in the form of a circular light spot of diameter D with a normal (according to Gaussian) distribution of illumination $\mathrm{E}$ in it. In this case, the illumination at an arbitrary point of a round light spot is determined by the formula:

$$
E(x, y)=E_{0} \frac{1}{\sigma \sqrt{2 \pi}} e^{-\frac{x^{2}+y^{2}}{2 \sigma^{2}}},
$$

where $E_{0}$ is the illumination at the center of the light spot, $\sigma$ is the Gaussian distribution parameter; $x, y$ are the coordinates of an arbitrary point in the Cartesian coordinate system with the origin in the center $\mathrm{O}$ light spot. Then the electrical signal-response from each photosensitive element of the matrix according to (11) is determined by the integral:

$$
I_{i, j}=k \int_{y 1_{i j}}^{y 2_{i j}} \int_{x 1_{i j}}^{x 2_{i j}} E_{0} \frac{1}{\sigma \sqrt{2 \pi}} e^{-\frac{x^{2}+y^{2}}{2 \sigma^{2}}} d x d y
$$

The periodicity and parity/oddness of the direction-finding characteristics $\mathrm{FSi}$ and $\mathrm{FSj}$ of the

If we consider the direction-finding characteristics of such a matrix as the dependence of the output electrical signals on the coordinates of the center of the light spot on the matrix, then it can be argued that these characteristics are described by periodic functions with a period corresponding to the spatial period of a matrix with a checkerboard structure:

$$
T=2(a+t)
$$

To study the direction-finding characteristics, it is sufficient to investigate the dependence of the output electrical signals from the matrix on the coordinates of the center of the light spot, which moves within the matrix area with dimensions $\mathrm{T} \times \mathrm{T}$.

For the functional representation of the directionfinding characteristics of the position-sensitive matrix of photosensitive elements, the obtained dependences are approximated by a known technique, for example, by a polynomial of two variables of degree 2 (n-1). Polynomials that interpolate the dependence of the direction-finding characteristics (3) of the matrix are graphically represented as a surface in the Cartesian rectangular coordinate system $\mathrm{O}$ xyz. In this case, the $\mathrm{x}$ coordinate of each surface point on the 3D graph corresponds to the $\mathrm{x}$ coordinate of the center of the light spot, the y coordinate to the y coordinate of the center of the light spot, and the $\mathrm{z}$ coordinate to the value of the corresponding dependence of the direction-finding characteristics of the matrix (the total electrical signal from the corresponding outputs of rows and columns of the matrix).

To increase the dynamic range of the optical coordinator, a fundamentally new topology of interconnections of light-sensitive semiconductor elements of a position-sensitive matrix was proposed by authors. The photosensitive elements of the proposed matrix can be made depending on the required spectral range based on $\mathrm{Si}, \mathrm{A} 3 \mathrm{~B} 5$ solid solutions, etc. An increase in the dynamic range is achieved since the light spot on the matrix surface is formed with a size of at least $2 \times 2$ matrix elements; to increase the speed, PIN, MPM, or MDP, avalanche photodetectors can be used as photosensitive elements. Photosensitive elements are 
organized in rows and columns according to the proposed topology (similar to the previous topology). The electrical signals from the elements of each row (column) are summed up, and the electrical signals from the elements of adjacent even (odd) rows (columns) are subtracted (Fig. 2).

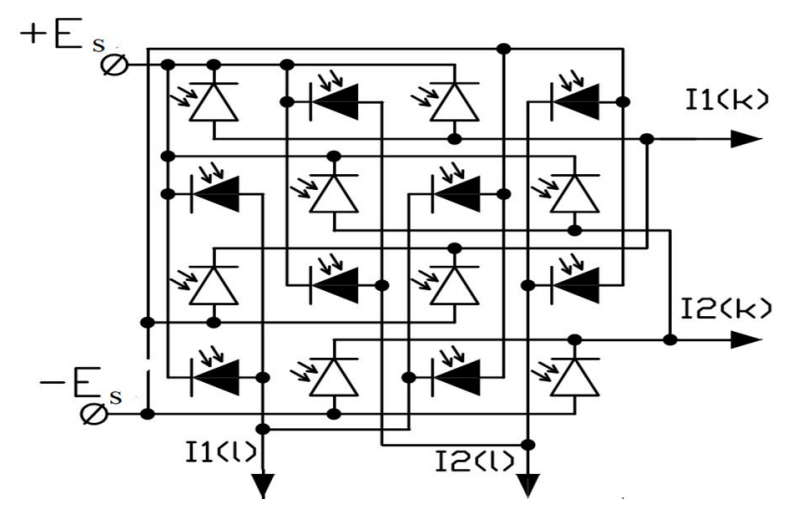

Fig. 2. Schematic diagram

of a position-sensitive array of photosensitive elements with an extended dynamic range

Due to this, electrical signals are generated at each of the matrix outputs, proportional to the difference in illumination of all photosensitive elements of adjacent even (odd) rows (columns) of such a matrix. Using the proposed interconnection topology makes it possible to determine simultaneously both coordinates of the light spot on one matrix of $\mathrm{N} \mathrm{x} \mathrm{M} \mathrm{elements} \mathrm{with} \mathrm{an} \mathrm{accuracy}$ of one matrix element. At the same time, for processing analog signals from a $4 \mathrm{Nx} 4 \mathrm{M}$ matrix, only $2 \mathrm{~N}+2 \mathrm{M}$ independent channels are enough to amplify the signal. To convert the received signals into a digital code, $4 \mathrm{Nx} 4 \mathrm{M}$ comparators and two encoders (respectively with $4 \mathrm{~N}$ and $4 \mathrm{M}$ inputs) are used. Note, that these same encoders easily solve the task of transforming these coordinates (for example, from coordinates relative to a matrix to coordinates relative to the carrier of this matrix).

\section{Conclusions}

The original position-sensitive matrix elements with a direction-finding characteristic comparable to a 4quadrant photodetector are proposed for the optical coordinator without application of the precise tracking electromechanical system for holding the light spot near the center of the photodetector element. In this case, there is also no need for the initial alignment of the optical coordinator with the analyzed beam. The matrix topology has made it possible to reduce significantly the number of processed analog signals from the photosensitive elements, which significantly simplifies the signal processing algorithm for determining the coordinates of the light.

\section{Conflict of Interest}

The authors claim that there are no possible financial or other conflicts over the work.

\section{References}

[1]M. Huber, "CCD and CMOS sensors", Observing Photons in Space ISSI Scientific Reports Series, ESA/ISSI. ISBN 978-92-9221-938-8, pp. 391-408, 2010.

[2]F. De Nisi, F. Comper, L. Gonzo, M. Gottardi, D. Stoppa, A. Simoni, J. A. Beraldin, "CMOS sensor optimized for laser spot position detection", IEEE Journal of Sensors, vol. 5, no. 6, pp. 1296-1304, 2005.

[3]X. Qi, "Design realization and characterization of a position sensitive detector for fast optical measurement", Optical engineering, vol. 45, no. 1, pp. 014402-1-5, 2006.

[4]F. Michael, "Very large area cmos active-pixel sensor for digital radiography", IEEE Transactions on Electron Devices, vol. 56, no. 11, pp. 2623-2631, 2009.

[5]J. Henry, J. Livingstone, "Optimizing the wavelength response in one-dimensional p-Si Schottky barrier optical PSDs”, Physica Status Solidi A, 208, pp. 1718--1725, 2011.

[6]Song H. X., Wang et al., "Design and performance analysis of laser displacement sensor based on position sensitive detector (PSD)", Journ. Phys, Conf. Series, vol. 48. pp. $217-222,2006$.

[7]W. Wang and I. J. Busch-Vishniac, "The linearity and sensitivity of lateral effect position sensitive devices-an improved geometry," Electron Devices, IEEE Transactions on, vol. 36, no. 11, pp. 2475-2480, 1989.

[8]H. Andersson, "Processing and characterization of a mos-type tetra lateral position sensitive detector with indium tin oxide gate contact," Sensors Journal, IEEE, vol. 8, no. 10, pp. 1704-1709, 2008.

[9]A. Dlugaszek, J. Jabczynski, J. Janucki, W. Skrzeczanowski, "Optoelectronic sensor of longitudinal and angular displacements", Semiconductor Physics, Quantum Electronics \& Optoelectronics, vol. 2, no. 3, pp. 71 - 73, 1999.

[10] L.P. Salles and D. W. de Lima Monteiro, "Designing the response of an optical quad-cell as position-sensitive detector," Sensors Journal, IEEE, vol. 10, no. 2, pp. 286-293, 2010.

[11]C. F. Kuang, "Analyzing characteristic of the fourquadrant detector applied to laser alignment", Optical Technique, vol. 30, no. 4, pp. 387-389, 2004.

[12] T. Ng, H. Tan, S. Foo, "Small gaussian laser beam diameter measurement using a quadrant photodiode," Optics \& Laser Technology, vol. 39, no. 5, pp. 1098-1100, 2007.

[13] Yu. Astapov, D. Vasiliev, Yu. Zalozhnev, "The theory of optoelectronic tracking systems", Moscow, 1988.

[14] A.Voronko, M.Moskalenko, G. Potapova, "Position-sensitive matrix of photosensitive elements for atomic force microscopy of nanoscale elements", VII International Russian-Ukrainian seminar "Nanophysics and Nanoelectronics”, St. Peterburg, 2006, pp. 95-96.

[15] A. Voronko, M. Moskalenko, G. Potapova, "Position-sensitive array of photosensitive elements with a record dynamic range", III International Conference on Optoelectronic Information Technologies "PHOTONICS-ODS 2005", Vinnytsia, pp. 201-202, 2005. 